\title{
Turbulent kinetic energy and self-sustaining tones: Experimental study of a rectangular impinging jet using high Speed 3D tomographic Particle Image Velocimetry
}

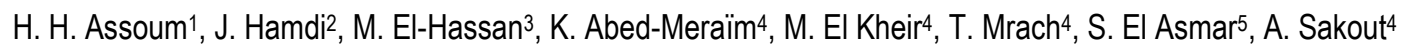 \\ ${ }^{1}$ Department of Mechanical Engineering, Beirut Arab University, Tripoli Campus, Lebanon. Phone: +9616218400; Fax: +961 6218400 \\ 2 Lebanese American University, Byblos, Lebanon. \\ ${ }^{3}$ Prince Mohamad Bin Fahed University, Saudi Arabia. \\ ${ }^{4}$ University of La Rochelle, LaSIE, France. \\ ${ }^{5}$ Institut Supérieur des Sciences Appliquées et Économiques, Tripoli, Lebanon.
}

\begin{abstract}
Impinging jets are widely used in ventilation systems to improve the mixing and diffusion of airflows. When a rectangular jet hits a slotted plate, an acoustic disturbance can be generated and self-sustained tones produced. Few studies have looked at the Turbulent Kinetic Energy (TKE) produced by the aerodynamic field in such configurations and in the presence of self-sustaining tones. The aim of this work is to investigate the energy transfer between the aerodynamic and acoustic fields generated in a rectangular jet impinging on a slotted plate. The present paper methodology is based on experimental data measurements using 3D tomographic Particle Image Velocimetry (PIV) technique and microphones. It was found that the spectrum of the TKE for $R e=5294$ (configuration of self-sustained tones) is $\mathrm{f}_{\mathrm{Re}}=5294=148 \mathrm{~Hz}$ which is smaller than that of the acoustic signal $\mathrm{F}_{0, \mathrm{Re}=5294}=164 \mathrm{~Hz}$. A negative peak of correlation $\mathrm{C}_{\mathrm{Re}=5294} \approx-0.24$ is obtained between the acoustic signal and TKE for $\mathrm{Re}=5294$. These results may lead to conclude that the acoustic cycle should be covered by the TKE period and the two signals of both fields are in opposition of phase in order to obtain an optimal configuration for energy transfer.
\end{abstract}

ARTICLE HISTORY

Revised: $3^{\text {rd }}$ Sept 2019

Accepted: $22^{\text {nd }}$ Sept 2019

\section{KEYWORDS}

Impinging jet;

Tomographic-Particle

Image Velocimetry;

turbulent kinetic energy;

self-sustaining tones.

\section{INTRODUCTION}

Impinging jets are encountered in many applications such as drilling [1] and ventilation and were widely studied numerically as in [2]. Ventilation systems in buildings are of vital importance for the provision of suitable air quality while meeting stringent energy requirements. Impinging jets are encountered in many ventilation systems, and these can have a major impact on the acoustic comfort and energy consumption [3-5]. In impinging jet, a flow coming from a jet exit hits an obstacle (perpendicular or at an angle). An impinging jet is crowded by vortices where fluid-structure interactions may be accompanied by a high level of acoustic generation due to the development of self-sustaining tones [6-9]. When a flow hits a wall, a "stress" on the fluid is created in the impingement contact area, generating a disturbance which travels upstream to the point where the vortices originated and regulates their detachment at the same frequency as that of the excitation produced at the obstacle.

Like the parameters of the flow, the geometry of the jet orifice or the nozzle plays a critical role in the production of self-sustaining tones [10-17]. Sondhauss et al. [18] showed that the Strouhal number relative to the frequency transmitted depends both on the distance between the jet outlet and the slotted plate, and on the speed of the jet, while remaining within the upper and lower limits of the velocity range that corresponds to the appearance of self-sustaining sounds. Chanaud et al. [19] noted the presence of pure and harmonic sounds in a laminar regime (between $\mathrm{Re}=1000$ and $\mathrm{Re}=$ 2500).

The acoustic field is related to the irrotational part of the velocity field. For low Mach number flows and a periodic acoustic field of period $T_{0}$, Howe's energy corollary [20] can be used to evaluate the acoustic power generated or absorbed in a flow using the vorticity, the velocity and the acoustic velocity which must be obtained using experimental data (difficult to access the acoustic velocity) or numerically. When a self-sustaining loop is optimized, energy transfer from the aerodynamic field to the acoustic field occurs in a geometric configuration that allows optimal interactions between the acoustic field and the aerodynamic fluctuations.

Many studies have been performed to study self-sustaining tones or to estimate the turbulence kinetic energy produced in 2D-PIV. Assoum et al. [21] used 2D PIV which leads to incomplete results because azimuthal component of velocity has an important effect in these configurations. Alekseenko et al. [22] also considered the Turbulent Kinetic Energy in an impinging jet and the authors calculated the dissipation term from instantaneous Stereo-PIV velocity fields in order to compare swirling and non-swirling impinging jets. A few studies, such as those by [23-25], considered the Turbulent Kinetic Energy in Tomographic-PIV velocity fields. In this work, we studied the Turbulent Kinetic Energy (denoted by 
TKE) in High Speed 3D Tomographic-PIV, and the acoustic signal was measured with a microphone. The main objective of this work was to investigate the optimal conditions for the transfer of energy from the aerodynamic to the acoustic field at different Reynolds numbers $(\mathrm{Re}=4632, \mathrm{Re}=5294$ and $\mathrm{Re}=5956)$. The Reynolds numbers selected had relatively high and low levels of acoustic generation with the presence of self-sustaining tones.

New insights about energy transfers were revealed. Higher amplitudes of fluctuations near the jet exit were obtained for high noise configurations what could facilitate the upstream propagation of the perturbation of control created at the impinging wall. The acoustic cycle was smaller than the TKE one, indicating adequate conditions energy exchange. This work should be very useful in understanding the energy transfer mechanism in such configurations, which will in turn contribute to the development of noise reduction techniques.

\section{EXPERIMENTAL METHOD}

\section{Airflow Facilities}

A jet impinging on a slotted plate was generated in the laboratory at a free stream velocity denoted by $U_{0}$. A $4 \mathrm{~mm}$ thick aluminium plate equipped with a $10 \mathrm{~mm}$ high slot was set up in order to achieve the configuration of a jet impinging on a slotted plate. The experimental setup of our study is shown in Figure 1. The subsonic airflow is produced by a SIEMENS compressor (type VFD : variable frequency drive in order to produce different Reynolds numbers) and then the airflow passes through a damping chamber before a $1250 \mathrm{~mm}$ long rectangular tube (equipped with a honey comb in order to reduce the flow fluctuations before it exit from the nozzle) which was prolonged by a rectangular convergent. Hence, the setup creates a free jet of $\mathrm{H}=10 \mathrm{~mm}$ high and a length $\mathrm{L}_{\mathrm{z}}=190 \mathrm{~mm}$ in $\mathrm{Z}$ direction. A $4 \mathrm{~mm}$ thick aluminum plate was fixed with a beveled slot of the same dimensions as the convergent outlet and perfectly aligned with the latter using a displacement system. The exit to plate distance is denoted by $\mathrm{L}$ (Where $\mathrm{L}=40 \mathrm{~mm}$ ). The ratio between $\mathrm{L}$ and $\mathrm{H}$, (confinement ratio) is $\frac{\mathrm{L}}{\mathrm{H}}=4$. The Reynolds number is based on the hydraulic height of the nozzle, $\mathrm{H}_{\mathrm{h}}$, the maximum stream-wise velocity at the jet exit $\mathrm{U}_{0}$ (obtained using PIV technique described below), and the kinematic viscosity of air.
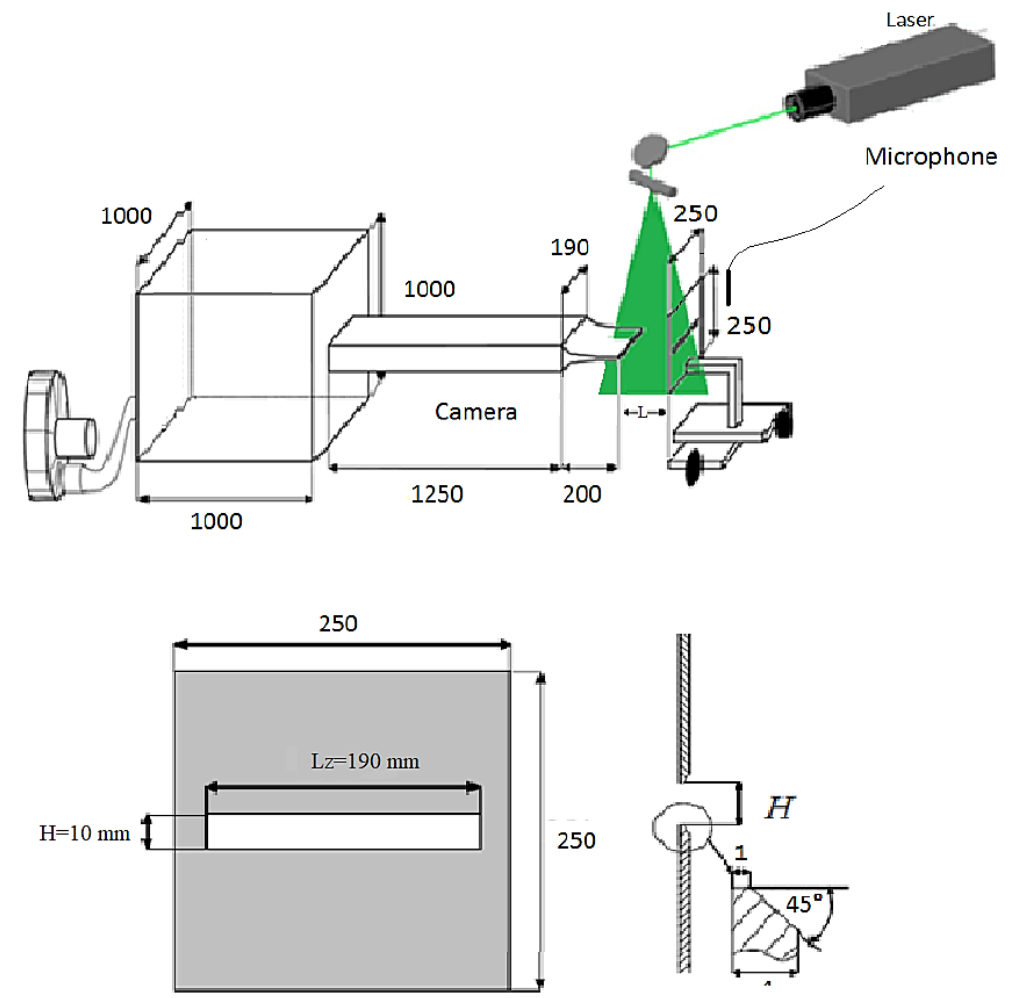

Figure 1. Experimental setup (all dimensions are in $\mathrm{mm}$ ).

\section{High Speed 3D Tomographic-PIV}

In order to study the characteristics of an impinging jet on a slotted plate for a confinement of $\frac{\mathrm{L}}{\mathrm{H}}=4$ and different Reynolds numbers in the presence of self-sustaining tones, a High Speed 3D Tomographic-PIV system was employed to measure the three velocity components. Time resolved Tomographic-PIV consists in cross-correlating two successive recorded images order to obtain the kinematic field.

A $527 \mathrm{~nm}$ Nd: YLF LDY 300 Litron laser was used at $30 \mathrm{~mJ}$ per pulse, extended with a $1800 \mathrm{~mm}$ laser arm. The laser arm was equipped with a volume generator formed by diverging lenses that generate a volume illumination. The volume 
measured in the present study was $40 \times 60 \times 10 \mathrm{~mm}^{3}$ following the reference system (x-axis, $y$-axis, $\mathrm{z}$-axis: see Figure 1). Three Phantom V711 cameras with $1280 \times 800$ pixels were used. Two cameras were placed on the right side of the jet exit, one above the other, and the third was placed on the left of the jet at the same level as camera 1 (see Figure 2). (See the effect of cameras configurations in [26]).

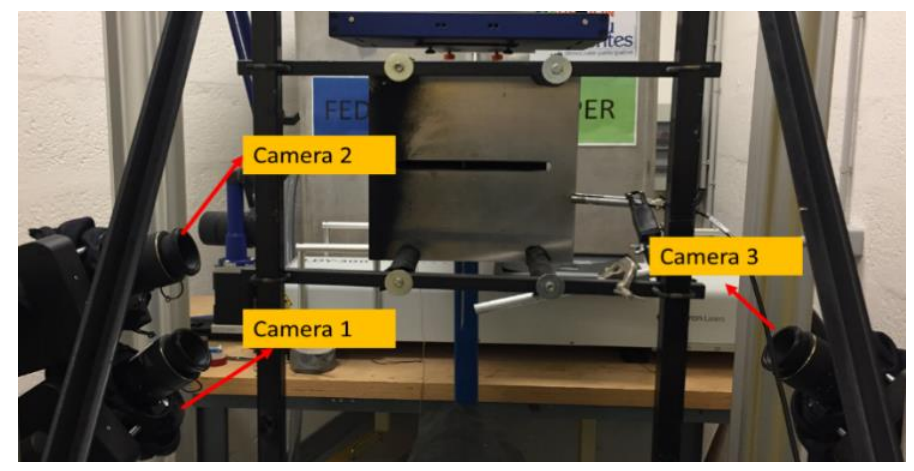

Figure 2. Cameras' Position for tomographic PIV.

The three cameras were placed $40 \mathrm{~cm}$ from the volume of interest located at the median plane between the nozzle and the plate. They were set up at an angle $\theta$ relative to the normal of the laser plane, which was chosen to optimize the quantity of light received. Moreover, they have a Scheimpflug arrangement that optimizes the sharpness of the particles. The views from the different cameras must be triangulated in order to obtain a tomographic reconstruction. Selfcalibration algorithm for tomographic PIV ([27] was developed to eliminate residual calibration discrepancies using recorded particle images). The volume self-calibration process requires two steps: in the first, the vector map of disparities is calculated, and in the second the calibration is corrected. These two steps must be repeated several times until the disparity (volume error) is less than 0.1 voxel in all sub volumes.

Particle Image Velocimetry (PIV) image pairs were acquired at a sampling frequency of $2 \mathrm{kHz}$. Once the particle density was optimized, the time interval $\Delta \mathrm{t}$ between the first and second exposure was adjusted so that the particles moved a maximum of about 7 to 10 pixels. Olive oil particles were seeded using a TOPAS SLG 270 condenser aerosol generator which produced particles of an average size of $4 \mu \mathrm{m}$ in diameter.

The self-calibration process consists in using the correspondence of $3 \mathrm{D}$ particles by triangulation. The residual triangulation error, called "disparity", is then used to update and correct the mapping functions of all the cameras. Calibration is carried out in two steps: first, the 3D mapping function is determined experimentally by recording images of the three-dimensional calibration pattern. Subsequently, the calibration map is refined by the self-calibration technique to minimize the disparity fields. This process reduces the calibration error to less than 0.1 voxel, which is acceptable for accurate tomographic reconstruction. Volumetric self-calibration was developed on the basis of calculating the 3D position of the corresponding particles by triangulation. The residual triangulation error ("disparity") is then used to correct the mapping functions of all the cameras. To summarize, the self-calibration of the volume is based on two successive stages (calculation of disparity vector and correction of the calibration). These two steps are repeated until a disparity of less than 0.1 voxel is obtained for all sub-volumes.

The three-dimensional distribution of the light scattered by the tracers (particles) is reconstructed in the form of a 3D matrix of voxels representing the luminous intensity of the object plane (physical space) from its projection on camera sensors. This reconstruction is carried out using MART ("Multiplicative Algebraic Reconstruction Technique"), which is an iterative technique, as already mentioned. The accuracy of reconstruction depends heavily on several factors, including the number of recording cameras and the density of recorded images. The kinematic field was reconstructed by the algorithm in the DaVis 8.3.0 software of LaVision that was used for our measurements.

The "calculation of correlation peaks" operation associated with the "tomographic PIV" module calculates the velocity vector field from the reconstructed volume. The reconstructed image distribution in the volume was analysed with an iterative multi-grid volume deformation scheme, attaining a final interrogation box size of 48 voxels.

\section{Acoustic Measurements}

The acoustic pressure was measured simultaneously with the velocity measurements. A microphone was placed behind the plate (away from the aerodynamic disturbances) to measure the sound pressure. The Brüel \& Kjær $1 / 2$ inch (type 4189) microphone used has a sensitivity range of $7 \mathrm{~Hz}$ to $20 \mathrm{kHz}$. For sound field measurements, the sampling frequency was $10 \mathrm{KHz}$. Microphone signals were processed with a B\&K Nexus amplifier/conditioner before being recorded via a National Instruments PXI-6224 acquisition board mounted on a National Instruments PXI 10441 data logger. This microphone has an uncertainty value of $0.2 \mathrm{~dB}$. 


\section{RESULTS AND DISCUSSION}

Self-sustaining tones may be accompanied by very high acoustic levels. The Sound Pressure Levels as function of Reynolds number and confinement ration $(\mathrm{L} / \mathrm{H})$ is given in Figure 3[28]. In this work $\mathrm{L} / \mathrm{H}=4$ is considered and the Sound Pressure Levels (SPL) obtained by the jet are shown in Table 1 as a function of the Reynolds number for $\frac{\mathrm{L}}{\mathrm{H}}=4$. The maximum streamwise velocity is denoted by $U_{0}$. The increase of $U_{0}$ from 7 to $8 \mathrm{~m} / \mathrm{s}$ shown in Figure 4(a) and (b) (from $\mathrm{Re}=4632$ to $\mathrm{Re}=5294$ ) resulted in an increase in the acoustic level from $90.8 \mathrm{~dB}$ to $97.3 \mathrm{~dB}$. A further increase of $\mathrm{U}_{0}$ from 8 to $9 \mathrm{~m} / \mathrm{s}$ shown in Figure 4(b) and (c) (from $\mathrm{Re}=5294$ to $\mathrm{Re}=5956$ ) was responsible for a decline in the acoustic level by $3.6 \mathrm{~dB}$, as shown in Table 1 (from $97.3 \mathrm{~dB}$ to $\sim 93.7 \mathrm{~dB}$ ). For each Reynolds number, self-sustaining tones were produced, as can be seen in Figure 5(b), Figure 6(b) and Figure 7(b) from the peak of frequency in the acoustic power spectrum. Actually, the acoustic level started to increase when the Reynolds number increased from $\operatorname{Re}=4632$ to a peak at $\mathrm{Re}=5294$. Thereafter, the acoustic level decreased despite an increase in the Reynolds number. This Reynolds number $(\operatorname{Re}=5294)$ is optimal for the feedback loop of the acoustic excitation, as shown by [20], and could promote energy transfer from the dynamic to the acoustic field. In this study, we considered three Reynolds numbers $(\operatorname{Re}=4632$, $\mathrm{Re}=5294$ and $\mathrm{Re}=5956$ ) in order to analyse the TKE in each configuration, before and after an acoustic peak, using High Speed 3D Tomographic-PIV measurements.

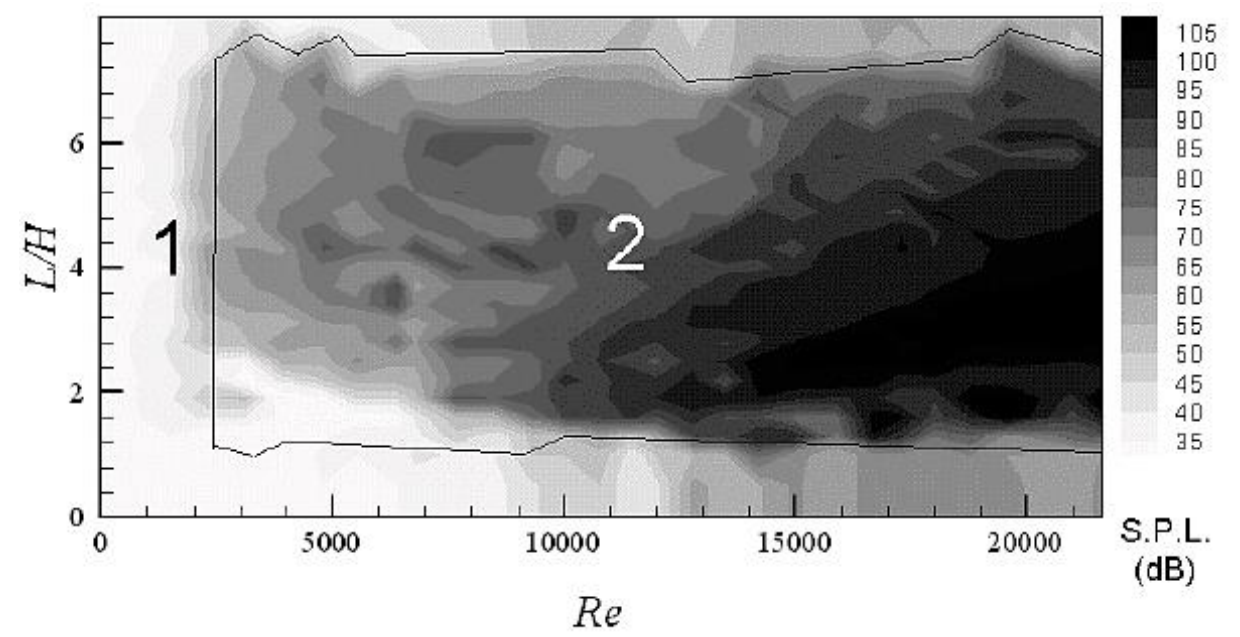

Figure 3. Acoustic level for different Re and L/H [25].

Table 1. Sound Pressure Level (SPL) as a function of the Reynolds number.

\begin{tabular}{cccc}
\hline SPL $(\mathrm{dB})$ & 90.8 & 97.3 & 93.7 \\
\hline $\operatorname{Re}$ & 4632 & 5294 & 5956 \\
\hline
\end{tabular}

Figure 5 to Figure 7 show the acoustic signals and their power spectra for $\mathrm{Re}=4632, \mathrm{Re}=5294$ and $\mathrm{Re}=5956$, respectively. The acoustic signal was periodical for $\mathrm{Re}=4632$ (Figure 5(a)) with one excited frequency (Figure 5(b)). Figure 6(a) indicates that the acoustic signal had a period displaying two successive peaks. The acoustic signal presented in Figure 7 (a) was less organised for $\mathrm{Re}=5956$. For $\mathrm{Re}=4632$, the fundamental frequency $\left(\mathrm{F}_{0}\right)$ of the acoustic signal was $\mathrm{F}_{0, \mathrm{Re}=4632}=204 \mathrm{~Hz}$ (Figure $5(\mathrm{~b})$ ). For $\mathrm{Re}=5294$ and $\mathrm{Re}=5956$ the fundamental frequency of the acoustic signal decreased and was almost the same, as shown in Figure 6 (b) and Figure 7(b) with $\mathrm{F}_{0, \mathrm{Re}=5294}=164 \mathrm{~Hz}$ (with a harmonic $2 * \mathrm{~F}_{0, \operatorname{Re}=5294}=328 \mathrm{~Hz}$ ) and $\mathrm{F}_{0, \operatorname{Re}=5956}=168 \mathrm{~Hz}$, respectively. 


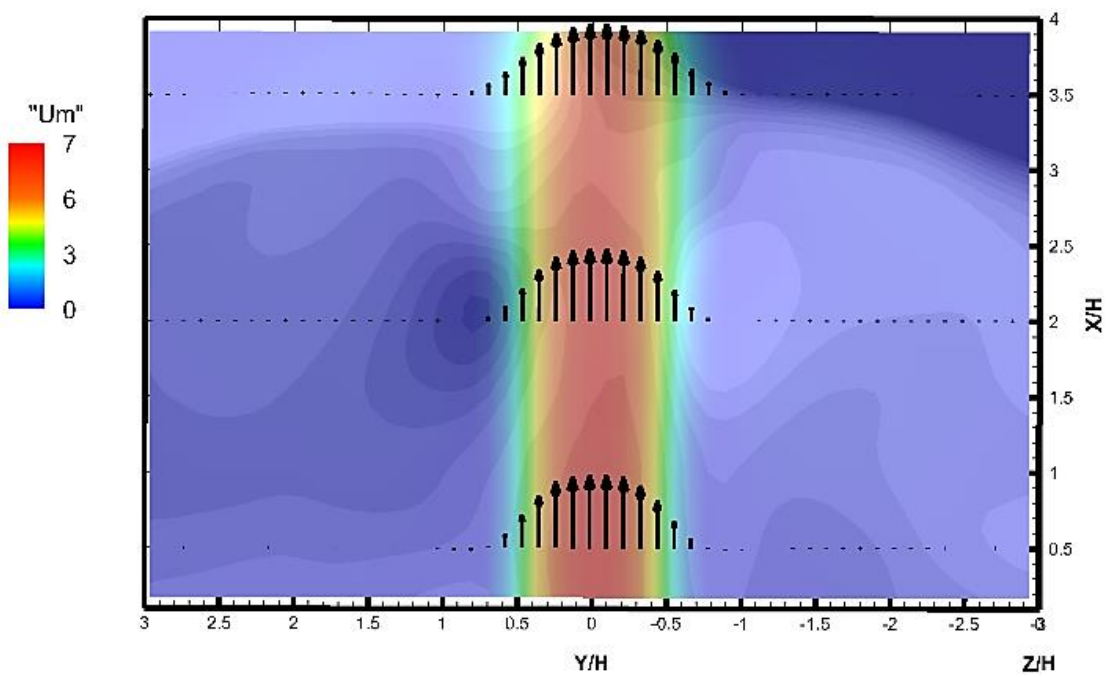

(a)

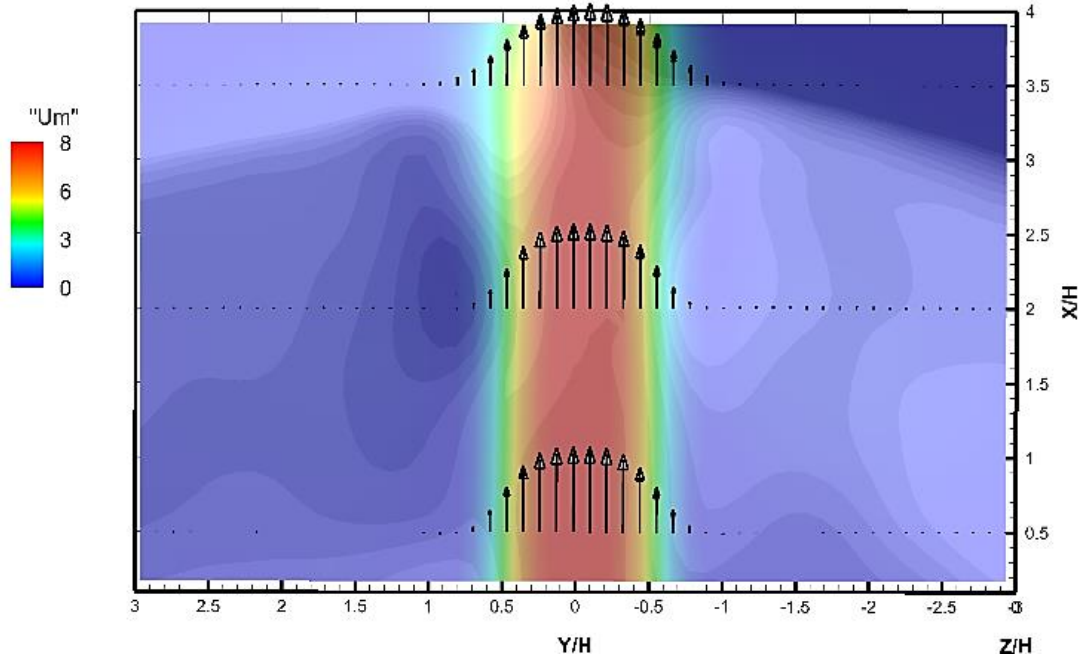

(b)

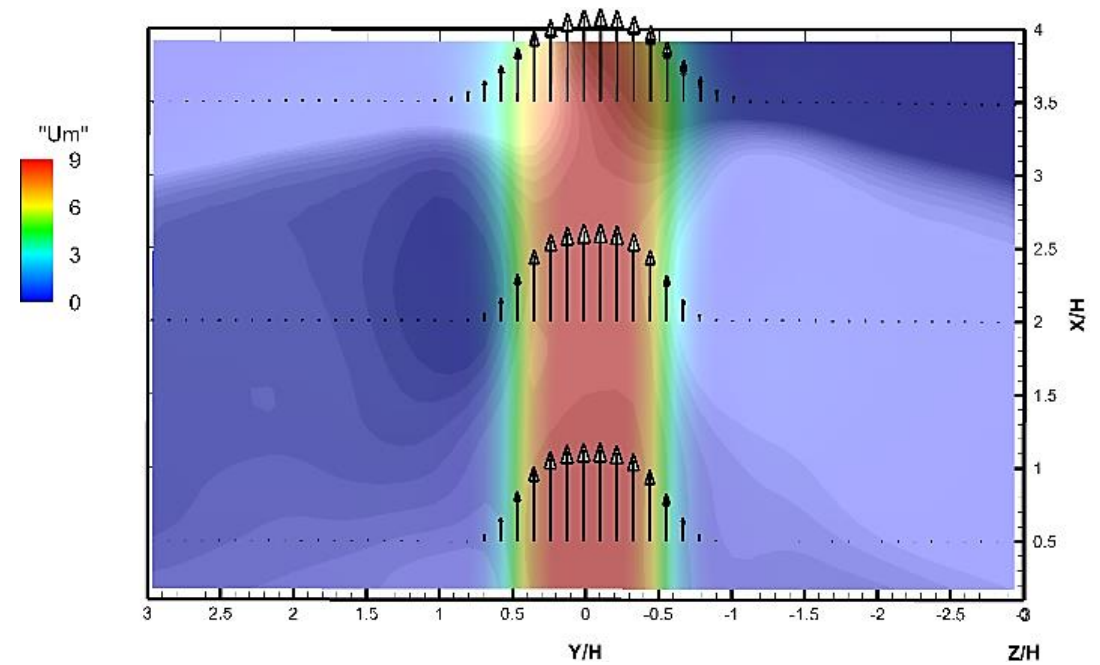

(c)

Figure 4. Mean streamwise velocity (color palette) in the central plane from Tomographic-PIV images superposed with mean transverse velocity (flood contour type) for: (a) $\mathrm{Re}=4632$, (b) $\mathrm{Re}=5294$ and (c) $\mathrm{Re}=5956$. 


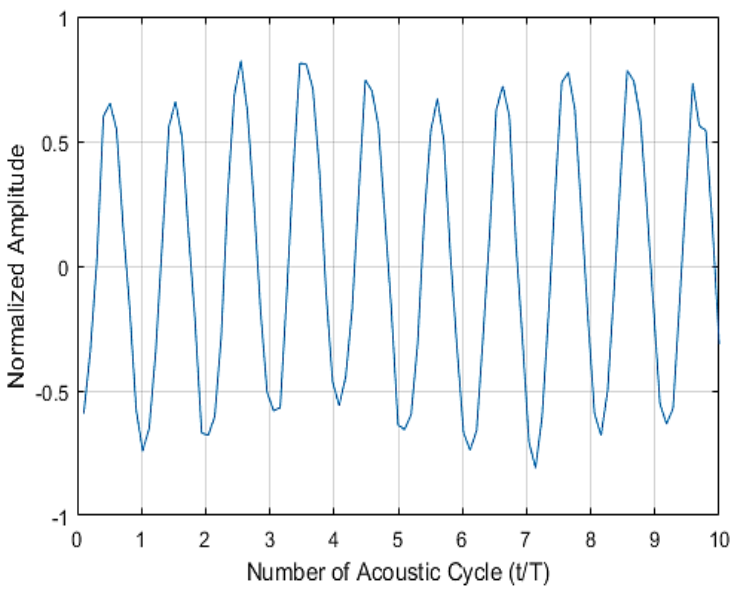

(a)

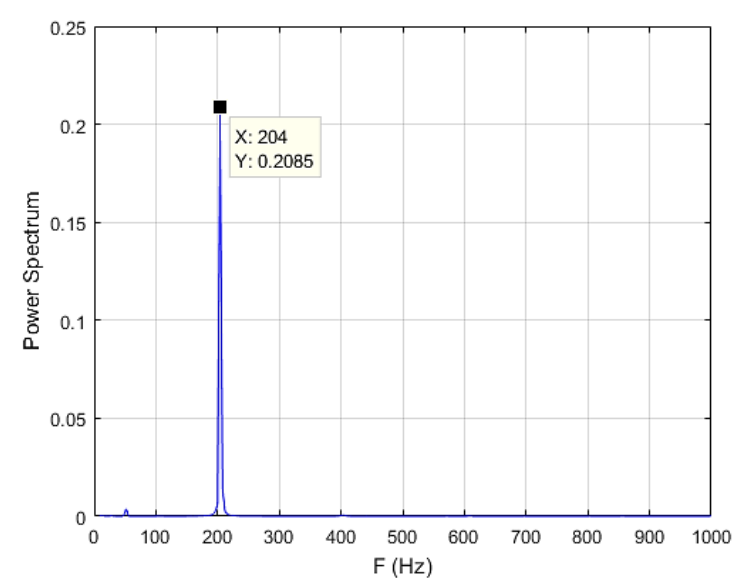

(b)

Figure 5. The acoustic signal and its spectrum: $\mathrm{Re}=4632$ and $\mathrm{L} / \mathrm{H}=4$.

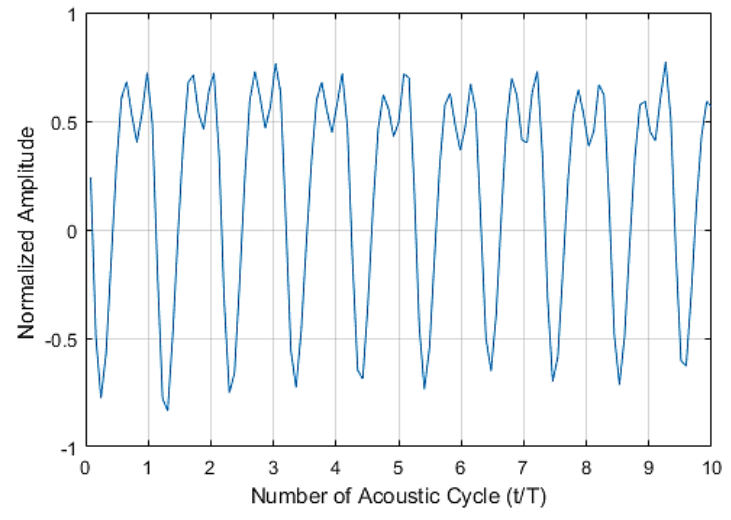

(a)

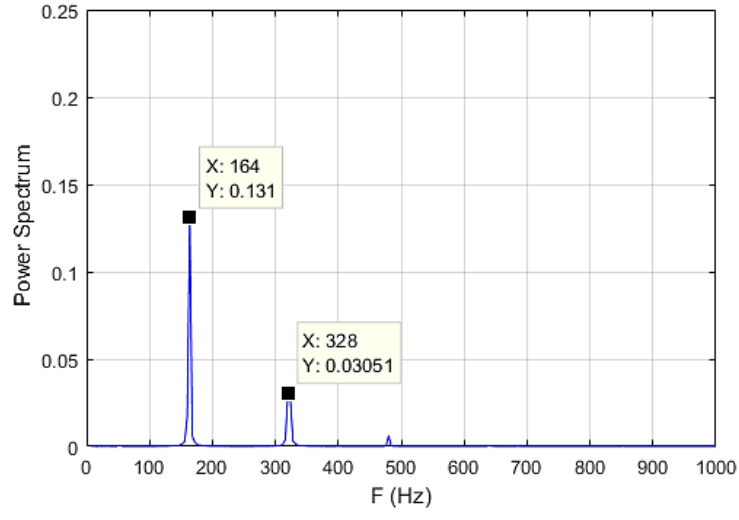

(b)

Figure 6. The acoustic signal and its spectrum: $\mathrm{Re}=5294$ and $\mathrm{L} / \mathrm{H}=4$.

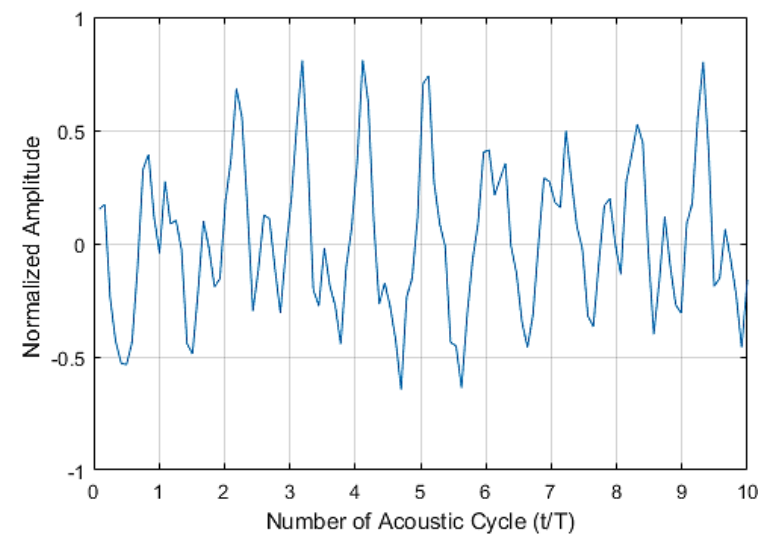

(a)

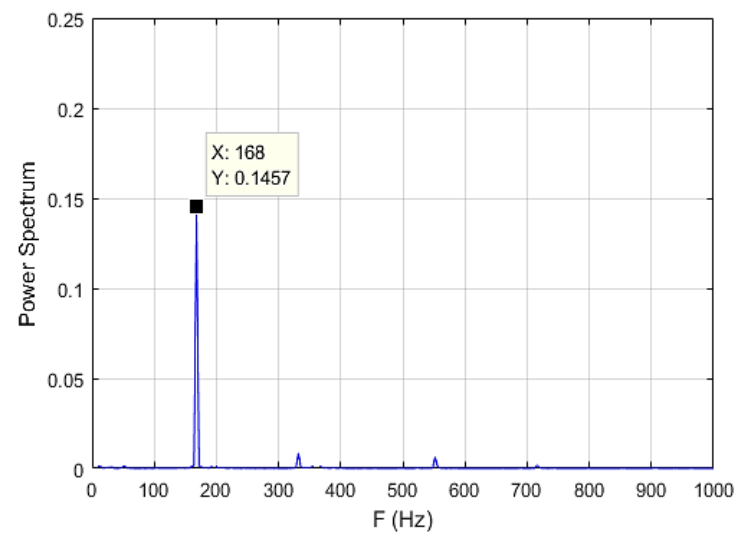

(b)

Figure 7. The acoustic signal and its spectrum: $\mathrm{Re}=5956$ and $\mathrm{L} / \mathrm{H}=4$.

Figure 7 presents the Root Mean Square components $V_{\text {rms }}$ and $W_{\text {rms }}$ for $R e=4632, R e=5294$ and $\operatorname{Re}=5956$. For the three Reynolds number, $\frac{V_{\mathrm{rms}}}{\mathrm{U}_{0}}$ started at 0.2 near the surface of impact.

As explained in the introduction, self-sustaining tones are created by a feedback loop that is sustained by an excitation which travels along the jet from the zone of vortex impact and reaches the jet exit, where the flow is very sensitive to any 
type of perturbation. This perturbation controls vortex generation and energy transfer between the aero-dynamic and acoustic fields.

Figure 8 shows that when $\left(\frac{Y}{H} \approx \mp 1.5\right)$ and near the jet exit $\left(0<\frac{\mathrm{X}}{\mathrm{H}}<1.5\right), \frac{\mathrm{W}_{\mathrm{rms}}}{\mathrm{U}_{0}}$ is greater, with higher levels ( Level $_{\text {maximum }} \approx 0.05$ ) for $\mathrm{Re}=5294$, which is an optimal configuration for self-sustaining tones [20]. For $\mathrm{Re}=4632$ and $\mathrm{Re}=5956$, there were lower levels of $\frac{\mathrm{W}_{\mathrm{rms}}}{\mathrm{U}_{0}}$ in this region of the jet (Level $\left.{ }_{\text {maximum }} \approx 0.03\right)$. These lower levels of $\frac{\mathrm{W}_{\mathrm{rms}}}{\mathrm{U}_{0}}$ in this region may be related to the propagation of the perturbation of control from the impact wall to the jet exit, and could explain why it was stronger when the self-sustaining tones were in optimal conditions, as for $\operatorname{Re}=5294$ (local peak of acoustic level, Table 1).

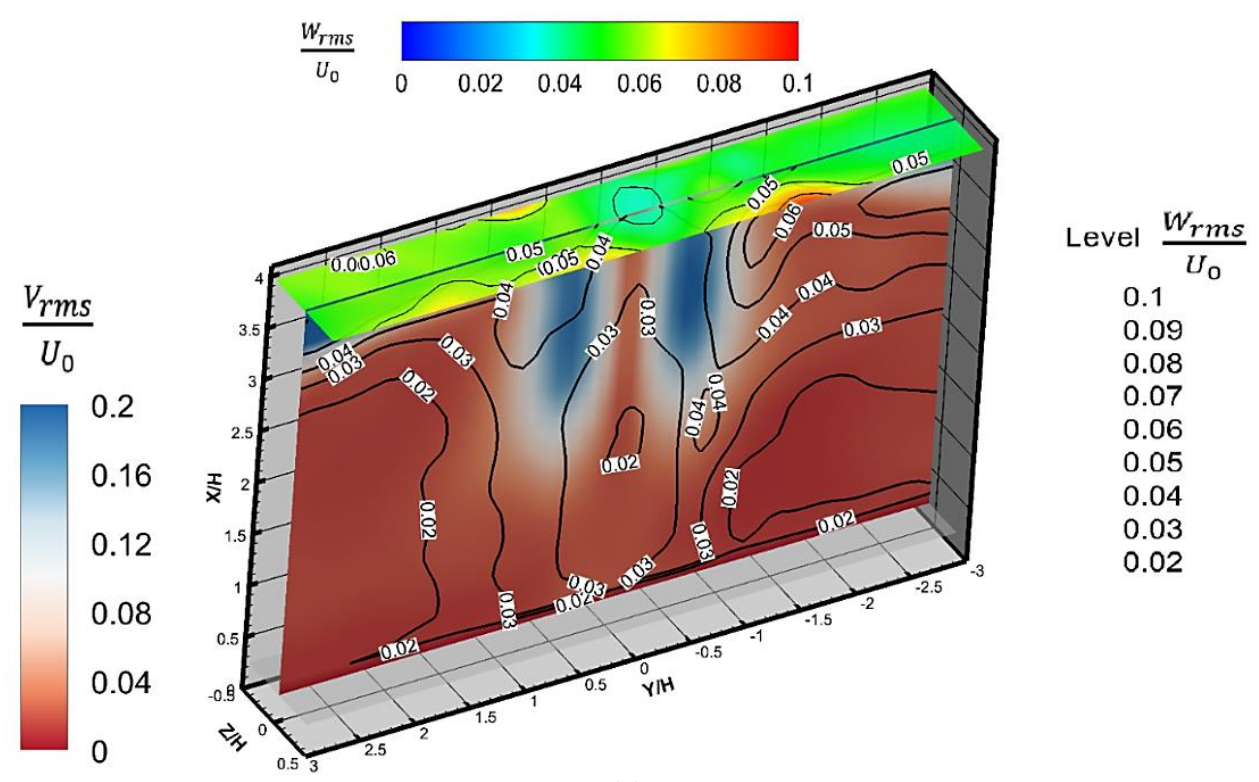

(a)

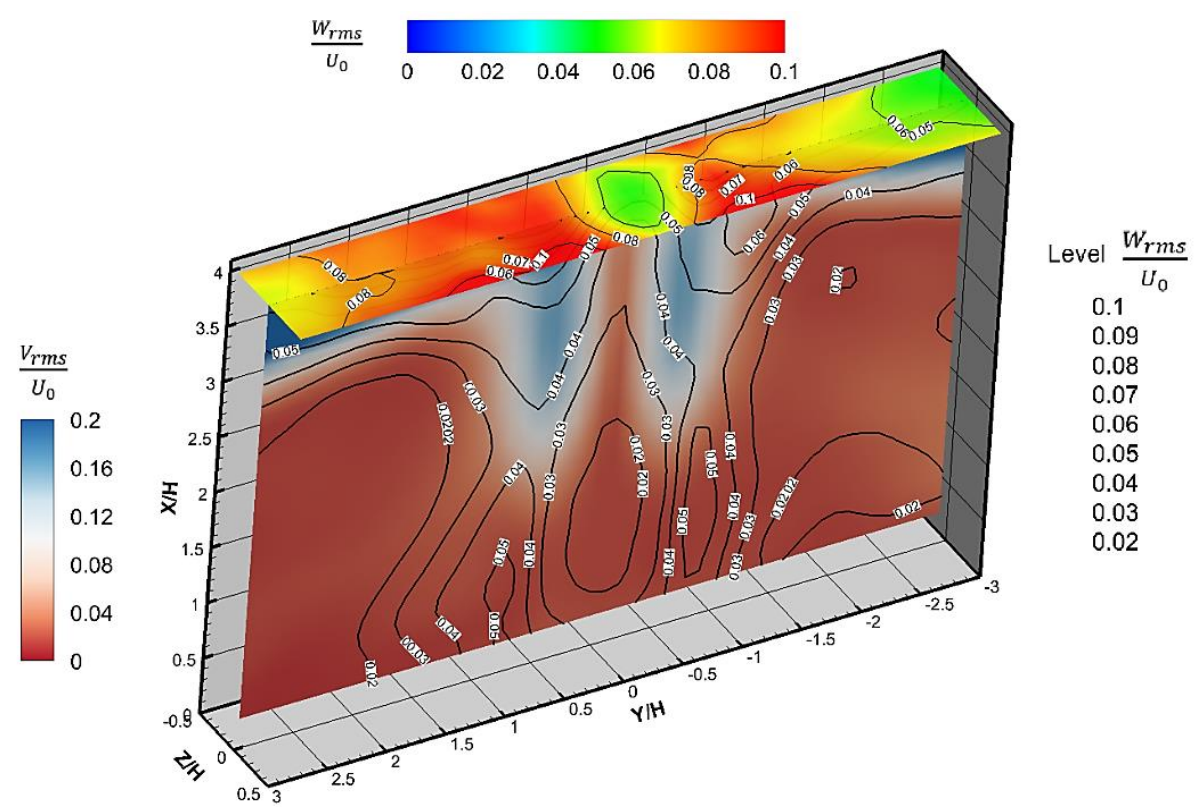

(b) 


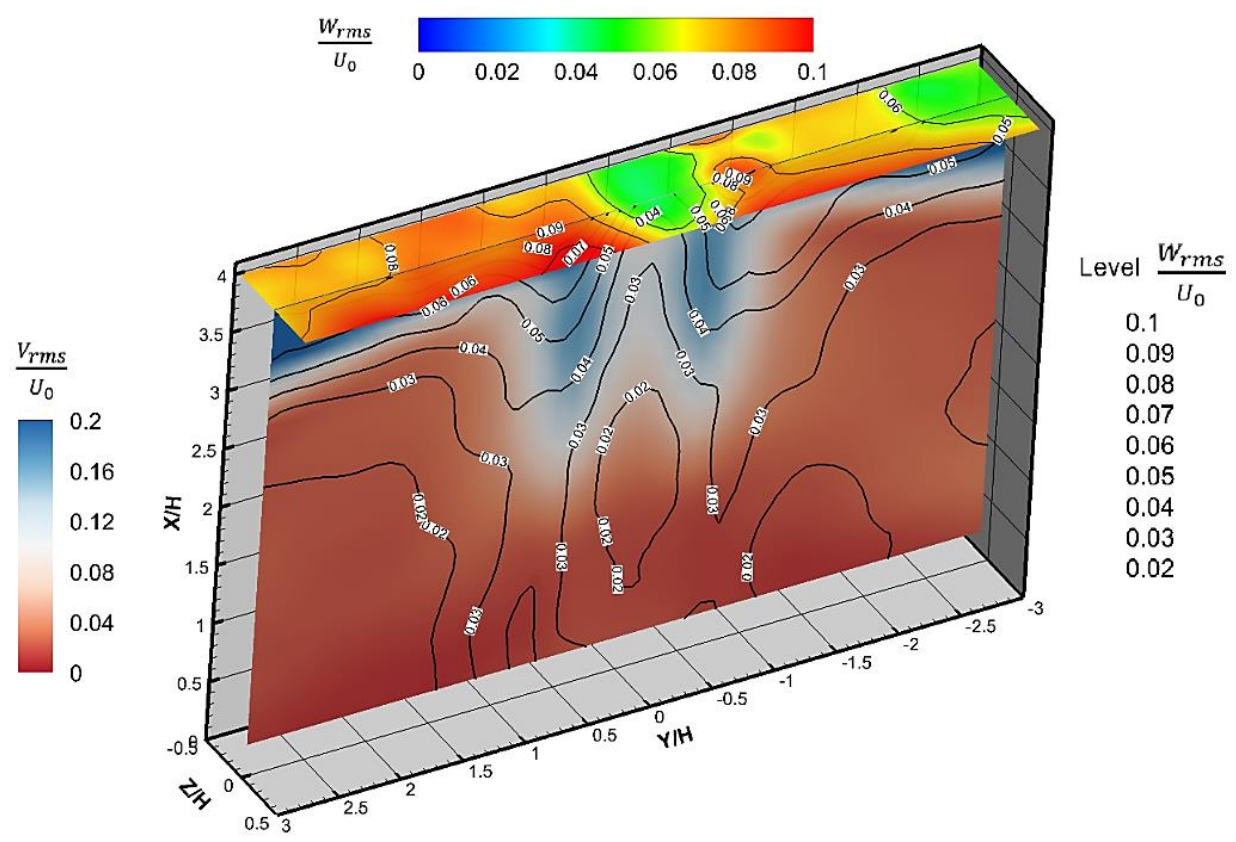

(c)

Figure 8. Root mean square components at the centre of the jet: (a) $\operatorname{Re}=4632$, (b) $\operatorname{Re}=5294$ and (c) $\operatorname{Re}=5956$.

Figure 9 illustrates the 3D iso-contours of the vorticity and the distribution of the average normalized TKE. As can be seen for $\mathrm{Re}=5294$ and $\mathrm{Re}=5956$ (Figure 9(b) and Figure 9(c)), TKE was highest near the surface of impact, where the deformation of vortices occurs. Moreover, there was a symmetric mode for $\mathrm{Re}=4632$ and $\mathrm{Re}=5345$. However, an antisymmetric mode was found for $\mathrm{Re}=5956$ (see the vorticity and velocity vectors). The change of aerodynamic mode (from symmetric to antisymmetric, as in our case) may be followed by a deterioration in the optimal conditions for energy transfer, which could be related to the local acoustic peak at $\mathrm{Re}=5294$.

The volumetric TKE (with TKE $=\frac{\mathrm{u}^{\prime 2}+\mathrm{v}^{\prime 2}+\mathrm{w}^{\prime 2}}{2}$ ) in Figure 9 was calculated on each voxel of the Tomographic-PIV images in a selected volume, where $\mathrm{U}, \mathrm{V}$ and $\mathrm{W}$ are the velocity components in $\mathrm{X}, \mathrm{Y}$ and $\mathrm{Z}$ directions, respectively $\left(U=U_{\text {mean }}+u^{\prime}, V=V_{\text {mean }}+v^{\prime}\right.$ and $\left.W=W_{\text {mean }}+w^{\prime}\right)$. The Turbulent Kinetic Energy (TKE) was normalized by the square of the maximum streamwise velocity $\mathrm{U}_{0}^{2}: \frac{\mathrm{TKE}}{\mathrm{U}_{0}^{2}}$. Therefore, the change as a function of time of TKE was calculated in the volume shown in Figure 9(b) in such a way that it contained the vortices which hit the wall, as described previously by [21]. Therefore, the sum of $\frac{\text { TKE }}{\mathrm{U}_{0}^{2}}$ in this volume, $\left(3.5<\frac{\mathrm{X}}{\mathrm{H}}<4,-1<\frac{\mathrm{Y}}{\mathrm{H}}<1\right.$ and $\left.-0.5<\frac{\mathrm{Z}}{\mathrm{H}}<0.5\right)$ was divided by the volume considered. By performing this calculation for successive PIV images, we obtained the normalized TKE per unit of volume as a function of time. Time was normalized by the acoustic period obtained from Figure 5, Figure 6 and Figure $7(\mathrm{~T}=1 / \mathrm{F})$; the volumetric normalized TKE was obtained as function of the acoustic period.

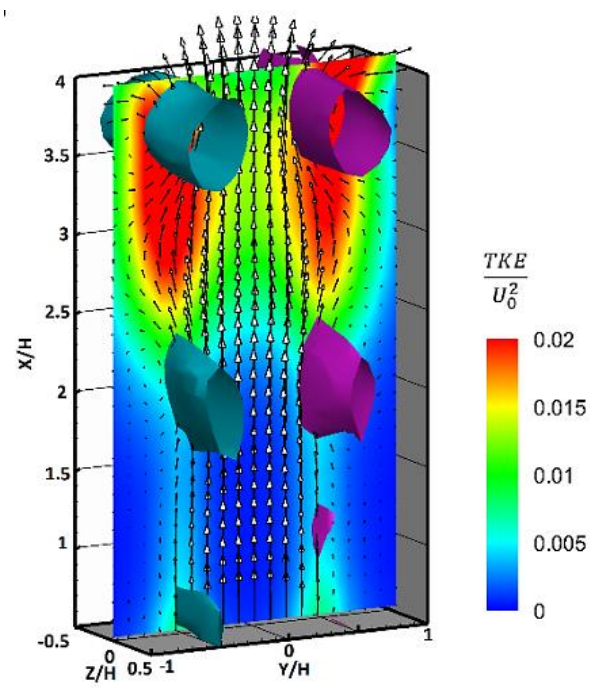

(a)

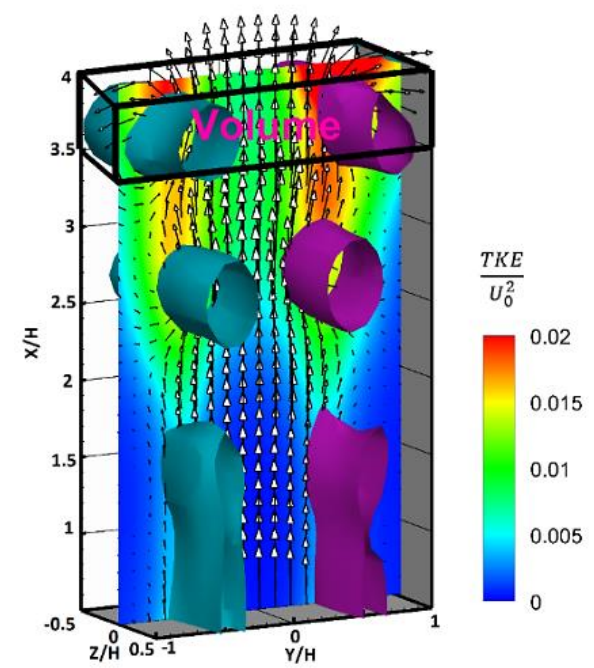

(b) 


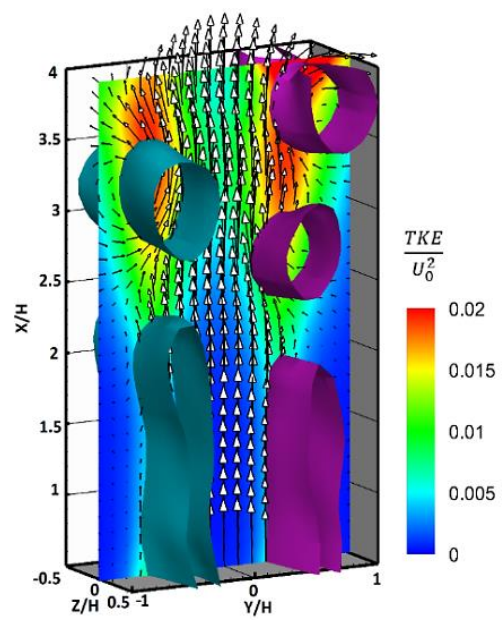

(c)

Figure 9. Vorticity 3D iso-contours and TKE field derived from Tomographic-PIV images at the centre of the jet: (a) $\mathrm{Re}=4632$, (b) $\mathrm{Re}=5294$ and (c) $\mathrm{Re}=5956$.

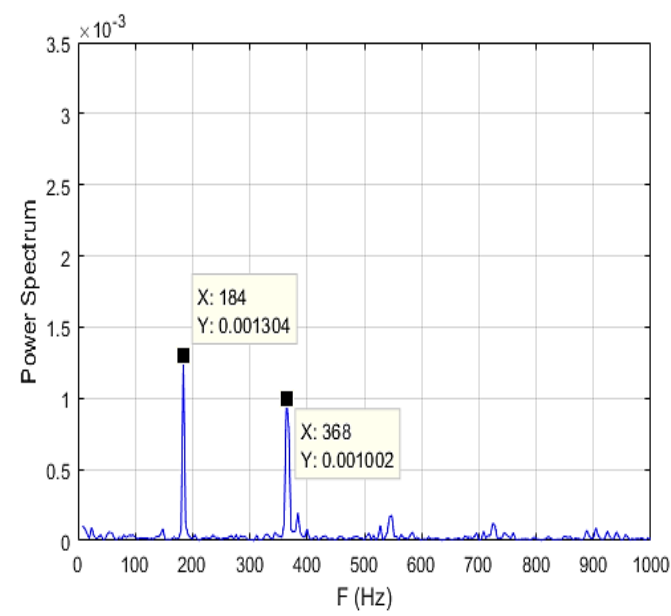

(a)

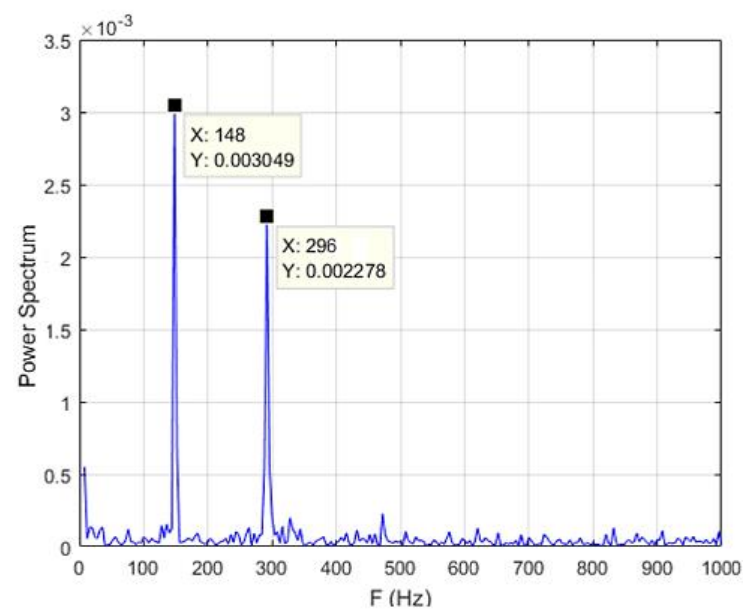

(b)

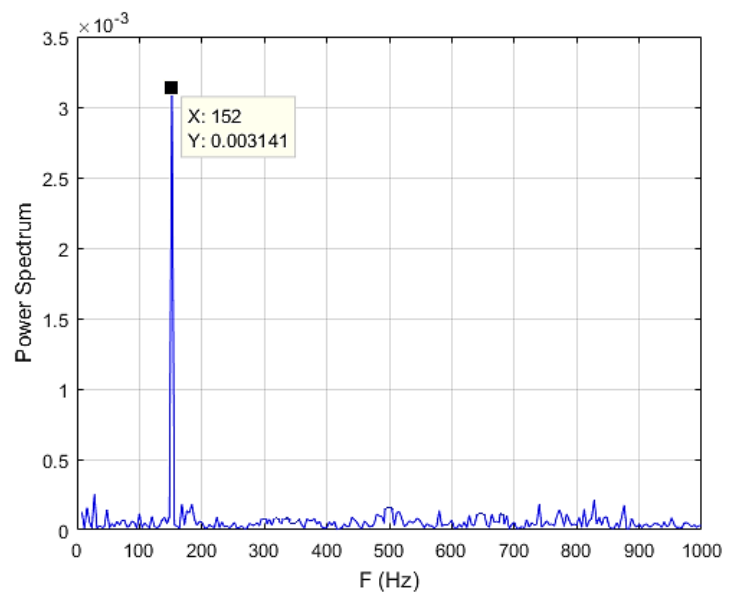

(c)

Figure 10. Power Spectrum of normalized TKE: (a) Re=4632, (b) $\operatorname{Re}=5294$ and (c) $\operatorname{Re}=5956$.

In the present work, 3D High Speed Tomographic-PIV was performed at a sampling rate of $2 \mathrm{KHz}$. The main flow vortices frequencies were below $0.4 \mathrm{KHz}$. Since TKE is very sensitive and can thus fluctuate a lot with time, we were mainly interested in the spectrum of this fluctuation and the frequencies that could be excited in the presence of self- 
sustaining tones. In the present work, 3D High Speed Tomographic-PIV was performed at a sampling rate of $2 \mathrm{KHz}$. The main flow vortices frequencies were below $0.4 \mathrm{KHz}$. Since TKE is very sensitive and can thus fluctuate a lot with time, we were mainly interested in the spectrum of this fluctuation and the frequencies that could be excited in the presence of self-sustaining tones. Figure 10 is a plot of the power spectra of TKE for each Reynolds number. The power spectra of the $\mathrm{TKE}$ for $\mathrm{Re}=4632, \mathrm{Re}=5294$ and $\mathrm{Re}=5956 \mathrm{had}$ peaks at $\mathrm{f}_{\mathrm{Re}=4632}=184 \mathrm{~Hz}, \mathrm{f}_{\mathrm{Re}=5294}=148 \mathrm{~Hz}$ and $\mathrm{f}_{\mathrm{Re}=5956}=$ $152 \mathrm{~Hz}$ and harmonics $2 * \mathrm{f}$ (for $\mathrm{Re}=4632$ and $\mathrm{Re}=5294$ ). Interestingly, all the excited frequencies were lower than the frequency of the acoustic signal $\mathrm{F}_{0, \mathrm{Re}=4632}=204 \mathrm{~Hz}$ for $\mathrm{Re}=4632$ (Figure 5), $\mathrm{F}_{0, \mathrm{Re}=5294}=164 \mathrm{~Hz}$ for $\mathrm{Re}=5294$ (Figure 6) and $\mathrm{F}_{0, \mathrm{Re}=5956}=168 \mathrm{~Hz}$ for $\mathrm{Re}=5956$ (Figure 7). This may indicate that self-sustaining tones occur when the period of TKE is larger than the period of the acoustic signal, enabling a better transfer of energy from the aerodynamic to the acoustic field.

In Figure 11, the cross-correlations between the acoustic signal and TKE for each Reynolds number are shown. For $\mathrm{Re}=5294$ (Figure 11(b)) the cross-correlation shows a negative peak delayed by $\frac{\mathrm{t}}{\mathrm{T}_{0}}=0.16$ with a correlation coefficient of $\mathrm{C}_{\mathrm{Re}=5294} \approx-0.24$. However, for $\mathrm{Re}=4632$ and $\mathrm{Re}=5956$, positive peaks of correlation were obtained with correlation coefficients of $\mathrm{C}_{\mathrm{Re}=4632}=0.18$ and $\mathrm{C}_{\mathrm{Re}=5956}=0.33$. The negative peak of correlation for the optimal self-sustaining tones configuration ( $\mathrm{Re}=5294$, local peak of the acoustic level) may suggest that both acoustic and TKE fields should be in opposition of phase for an optimal transfer of energy.

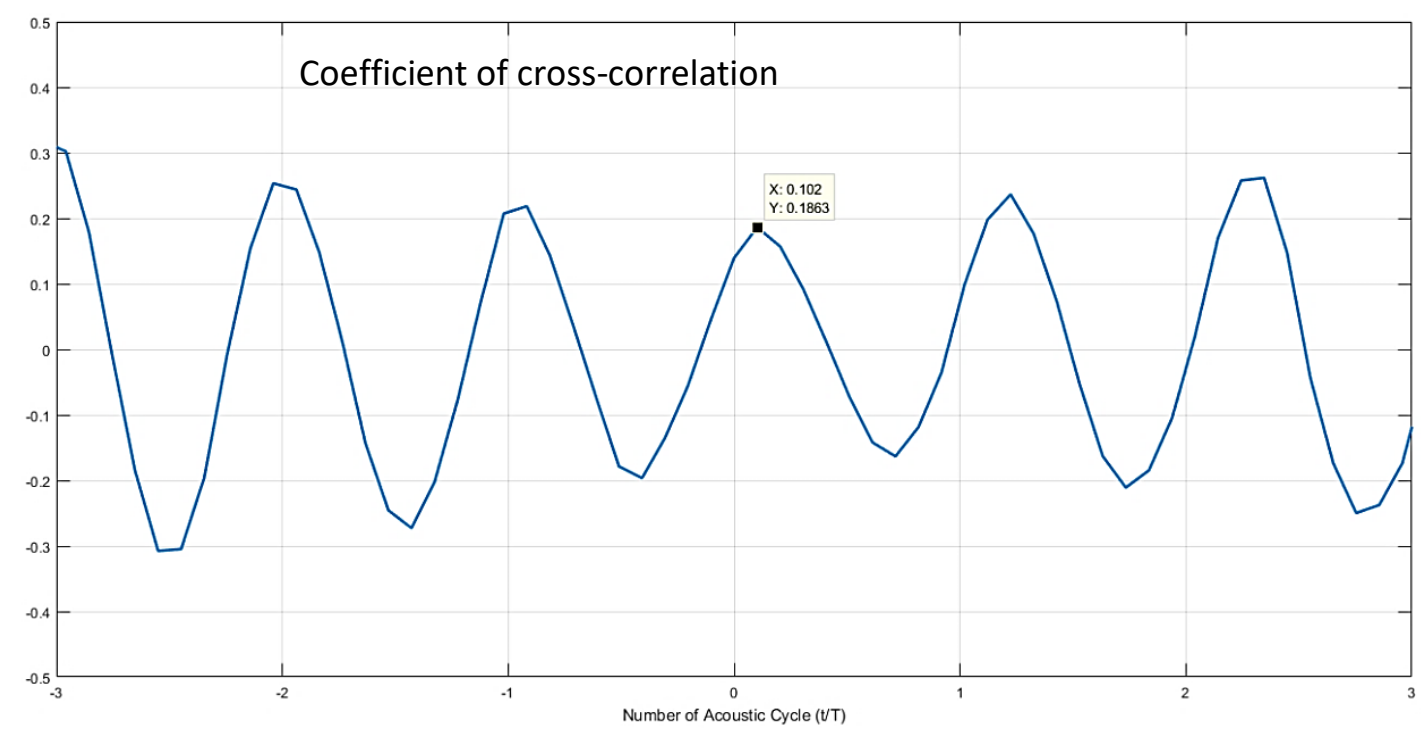

(a)

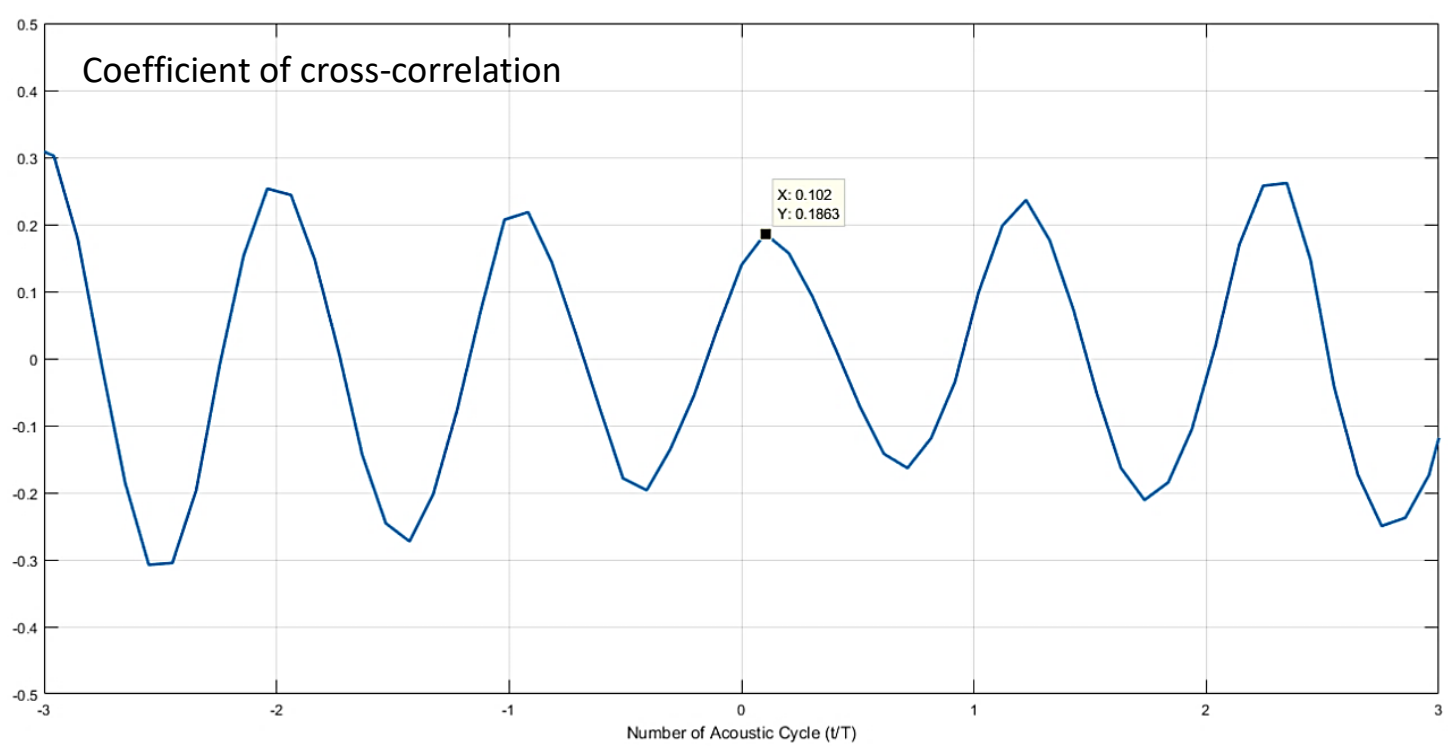

(b) 


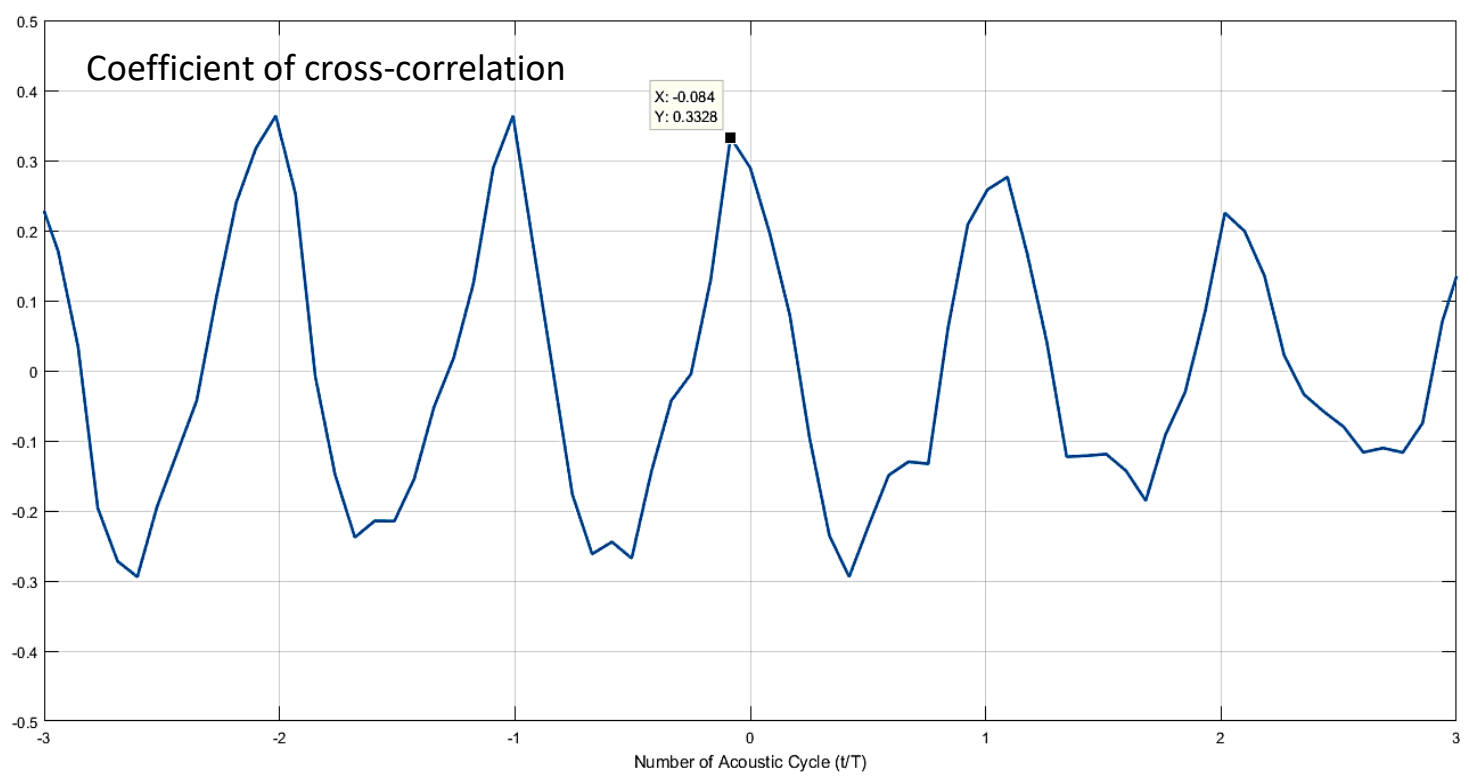

(c)

Figure 11. Cross-correlation of $\frac{\text { TKE }}{\mathbf{U}_{\mathbf{0}}^{2} * \text { Volume }}$ with the acoustic signal: (a) $\operatorname{Re}=4632$, (b) $\operatorname{Re}=5294$ and (c) $\operatorname{Re}=5956$.

\section{CONCLUSIONS}

The Turbulent Kinetic Energy (TKE) in a rectangular jet impinging on a slotted plate and the acoustic field generated were investigated experimentally using High Speed 3D Tomographic-PIV and a microphone. Three Reynolds numbers ( $R e=4632, R e=5294$ and $R e=5956$ ) with optimal and less optimal configurations for self-sustaining tones were considered. It was found that, in the case of an optimal configuration for self-sustaining tones $(\operatorname{Re}=5294$, local peak of the acoustic level), $W_{\text {rms }}$ presented higher amplitudes near the jet exit region. The change in 3D representation of the TKE as a function of time was calculated and its spectrum was analysed in order to detect if any frequencies were excited in the presence of self-sustaining tones. It was found that the spectrum of TKE had peaks of frequencies such that the period of the acoustic signal was smaller than that of the TKE for the three Reynolds numbers with self-sustaining tones. We also obtained a negative peak of correlation between the acoustic signal and TKE for $\mathrm{Re}=5294$ (a configuration with a local peak of the acoustic level).

These results may reveal that higher amplitudes of azimuthal fluctuations near the jet exit region exists in case of optimal self-sustained tones (high acoustic level) what facilitate the upstream propagation of the perturbation of control created at the impinging wall. The acoustic cycle was found to be smaller than the TKE one, which may indicate adequate conditions for the transfer of energy from the aerodynamic to the acoustic field. Negative correlation may suggest that the acoustic and TKE fields should not be in phase in order to obtain an optimal configuration for energy transfer. All these insights could be very useful for the reduce of the generation of noise in many applications of ventilation by controlling its energy source which feed the acoustic field.

Further work needs to be done using the phase average technique to calculate TKE with different Reynolds numbers. This may provide more information regarding the phase between the TKE and the acoustic field.

\section{ACKNOWLEDGEMENTS}

The authors wish to thank the region of Nouvelle Aquitaine for its financial support.

\section{REFERENCES}

[1] Deepak D, Ashwin Pai K. Study on abrasive water jet drilling for graphite filled glass/epoxy laminates. Journal of Mechanical Engineering and Sciences. 2019;13(2):5126-5136.

[2] Saeid NH, Busahmin BS, Khalid AA. Mixed convection jet impingement cooling of a moving plate. Journal of Mechanical Engineering and Sciences. 2019;13(3): 5528-5541.

[3] Assoum HH, El Hassan M, Abed-Meraïm K, Martinuzzi R, Sakout A. Experimental analysis of the aero-acoustic coupling in a plane impinging jet on a slotted plate. Fluid Dynamics Research. 2013;45:045503.

[4] Assoum HH, El Hassan M, Abed-Meraim K, Sakout A. The vortex dynamics and the self sustained tones in a plane jet impinging on a slotted plate. European Journal of Mechanics-B/Fluids. 2014;48:231-5. 
[5] Ziada S. Interaction of a jet-slot oscillator with a deep cavity resonator and its control. Journal of Fluids and Structures. 2001;15:831-43.

[6] Desmarais G, Rocha J. Discrete tones in subsonic jet engine test cells. Progress in Aerospace Sciences.2018;101:49-60

[7] Fei-Bin H, I-CheHsu, Jiann-MinHuang. Evolution of coherent structures and feedback mechanism of the plane jet impinging on a small cylinder. Journal of Sound and Vibration.2004;278: 1163-1179

[8] Karn LS, Con JD, Richard MK.The effect of a cavity on airfoil tones. Journal of Sound and Vibration. 2014;333: 1913-1931

[9] Didier V, Pierre B, André G. Self-sustained oscillations of a confined impinging jet. Flow, Turbulence and Combustion. 2007;78:1

[10] Nabil K, Lyes K, Mohamed A. Flow dynamics of an impinging plane jet generating slot tones. ICEMAEP. 2016

[11] Mrach T, Hamdi J, Abed-Meraim K, Alkheir M, Assoum H, Sakout A. Experimental analysis of the influence of the impinged plate by an impinging jet on the vortex dynamics using PIV. MATEC Web of Conferences. 2019;261:03005.

[12] Alkheir M, Assoum H, Abed-Meraim K, Mrach T, Hamdi J, Elsoufi L, Skaf H, Sakout A. Experimental investigation of the correlation between the dynamics of an impinging jet on a slotted plate and the acoustic field generated. MATEC Web of Conferences. 2019;261:03003.

[13] Hamdi J, Abed-Meraim K, Assoum H, Sakout A, Al-Kheir M, Mrach T, Rambault L, Cauet S, Etien E. Tomographic and TimeResolved PIV measurement of an Impinging Jet on a Slotted Plate. MATEC Web of Conferences. 2019;261:03004.

[14] Hamdi J, Abed-Meraim K, Assoum H, Sakout A, Al-Kheir M, Mrach T, Rambault L, Cauet S, Etien E. Volumetric Proper Orthogonal Decomposition of an impinging jet using SPIV measurement. MATEC Web of Conferences. 2019;261:03006.

[15] Assoum H, Hamdi J, Abed-Meraïm K, El Hassan M, Ali M, Sakout A. Correlation between the acoustic field and the transverse velocity in a plane impinging jet in the presence of self-sustaining tones. Energy Procedia. 2017;139:391-397.

[16] Anilkumar Hanchinal, Vadiraj Katti. Effect of orifice geometry and orifice to test section spacing on distribution of wall static pressure on a convex surface. Journal of Mechanical Engineering and Sciences. 2019;13(2):4835-4845.

[17] Hamdi J, Assoum H, Abed-Meraïm K, Sakout A. Volume reconstruction of a plane jet impinging on a slotted plate using the phase averaging method. Energy Procedia. 2017;139:404-409.

[18] Sondhauss C. Ueber die beim Ausströmen der Luft entstehenden Töne. Annalen der Physik. 1854;167:214-40.

[19] Chanaud R, Powell A. Some experiments concerning the hole and ring tone. The Journal of the Acoustical Society of America. 1965;37:902.

[20] Hourigan K, Welsh M, Thompson M, Stokes A. Aerodynamic sources of acoustic resonance in a duct with baffles. Journal of Fluids and Structures. 1990;4:345-70.

[21] Assoum H, Hamdi J, Abed-Meraïm K, El Hassan M, Hammoud A, Sakout A. Experimental investigation the turbulent kinetic energy and the acoustic field in a rectangular jet impinging a slotted plate. Energy Procedia. 2017;139:398-403.

[22] Alekseenko SV, Bilsky AV, Dulin VM, Markovich DM. Experimental study of an impinging jet with different swirl rates. International Journal of Heat and Fluid Flow. 2007;28:1340-59.

[23] Schäfer L, Dierksheide U, Klaas M, Schröder W. Investigation of dissipation elements in a fully developed turbulent channel flow by tomographic particle-image velocimetry. Physics of Fluids. 2011;23:035106.

[24] Schneiders JFG, Scarano F, Elsinga GE. Resolving vorticity and dissipation in a turbulent boundary layer by tomographic PTV and VIC+. Exp Fluids. 2017;58:27.

[25] Tokgoz S, Elsinga GE, Delfos R, Westerweel J. Spatial resolution and dissipation rate estimation in Taylor--Couette flow for tomographic PIV. Exp Fluids. 2012;53:561-83.

[26] Elsinga GE, Scarano F, Wieneke B, van Oudheusden BW. Tomographic particle image velocimetry. Exp Fluids. 2006;41:93347

[27] Wieneke B. Volume self-calibration for 3D particle image velocimetry. Exp Fluids. 2008;45:549-56

[28] Billon A, Valeau V, Sakout A. Two feedback paths for a jet-slot oscillator. Journal of Fluids and Structures.2005; 21(2):121132. 